\title{
Fluctuations of the front in a stochastic combustion model
}

\author{
Francis Comets $^{\mathrm{a}, *, 1,2}$, Jeremy Quastel ${ }^{\mathrm{b}, 3}$, Alejandro F. Ramírez ${ }^{\mathrm{c}, 2,4}$ \\ ${ }^{a}$ Laboratoire de Probabilités et Modèles Aléatoires, Université Paris 7-Denis Diderot, 2, Place Jussieu, 75251 Paris cedex 05, France \\ ${ }^{\mathrm{b}}$ Departments of Mathematics and Statistics, University of Toronto, 40 St. George Street, Toronto, Ontario M5S 1L2, Canada \\ ${ }^{\mathrm{c}}$ Facultad de Matemáticas, Pontificia Universidad Católica de Chile, Vicuña Mackenna 4860, Macul, Santiago, Chile
}

Received 3 November 2005; accepted 3 January 2006

Available online 5 October 2006

\begin{abstract}
We consider an interacting particle system on the one-dimensional lattice $\mathbf{Z}$ modeling combustion. The process depends on two integer parameters $2 \leqslant a \leqslant M<\infty$. Particles move independently as continuous time simple symmetric random walks except that (i) when a particle jumps to a site which has not been previously visited by any particle, it branches into $a$ particles, (ii) when a particle jumps to a site with $M$ particles, it is annihilated. We start from a configuration where all sites to the left of the origin have been previously visited and study the law of large numbers and central limit theorem for $r_{t}$, the rightmost visited site at time $t$. The proofs are based on the construction of a renewal structure leading to a definition of regeneration times for which good tail estimates can be performed.
\end{abstract}

๑ 2006 Elsevier Masson SAS. All rights reserved.

\section{Résumé}

On considère un système de particules en interaction sur $\mathbf{Z}$ modélisant les particules incandescentes d'un mécanisme de combustion. Le processus dépend de deux paramètres entiers $2 \leqslant a \leqslant M<\infty$. Les particules se déplacent indépendamment selon des promenades aléatoires simples symétriques à temps continu, mises à part les interactions suivantes : 1-quand une particule saute vers un site qui n'a jamais encore été visité, elle branche et fait place à $a$ particules; 2-quand une particule saute vers un site abritant $M$ particules, elle disparait. On démarre d'une configuration où seuls les sites à gauche de l'origine ont déja été visités et on étudie la loi des grands nombres et le théorème de la limite centrale pour $r_{t}$, la position du site le plus à droite visité à l'instant $t$. Les preuves reposent sur la construction d'une structure de renouvellement associée à des temps de régénération dont les queues peuvent être convenablement estimées.

(c) 2006 Elsevier Masson SAS. All rights reserved.

MSC: primary 82C22, 82B41; secondary 82B24, 60K35, 60G99

Keywords: Regeneration times; Interacting particle systems; Random walks in random environment

\footnotetext{
* Corresponding author.

E-mail addresses: comets@math.jussieu.fr (F. Comets), quastel@math.toronto.edu (J. Quastel), aramirez@mat.puc.cl (A.F. Ramírez).

1 Partially supported by CNRS, UMR 7599.

2 Partially supported by ECOS-Conycit grant CO5EO2.

3 Partially supported by NSERC, Canada.

4 Partially supported by Fondo Nacional de Desarrollo Científico y Tecnológico grant 1020686.
} 


\section{Introduction}

The method of regeneration times has been very successfully applied to problems of random walk in random environment (for example see $[16,17,6]$ ); in a one-dimensional setting it was already used via a renewal structure in [9].

In this article, we extend the method of regeneration times to the study of front propagation in an interacting particle system. The system we consider is a one-dimensional stochastic model of combustion. Heat particles move by symmetric nearest neighbor random walks on sites $\{x \in \mathbb{Z}: x \leqslant r\}$ of the integer lattice, with $r$ representing the position of a flame front. To the right of the flame front is a propellant. The first heat particle which reaches the propellant at $r+1$ immediately branches into $a \geqslant 2$ particles, and the front moves one step to the right. We also include an upper bound $M$ on the number of particles at each site, so that if a particle tries to jump to a site with $M$ particles, it is immediately killed. We show that the front in the model moves ballistically to the right, or, more precisely, prove a law of large numbers for $r_{t}$. The next question one might ask is of the fluctuations about the law of large numbers. We prove here that these are Gaussian, with a central limit theorem for $t^{-1 / 2}\left(r_{t}-v t\right)$.

For $M=\infty$ a discrete time version of the system we are considering has appeared in the literature under the enigmatic name "frog model", and laws of large numbers for the position of the front were proved, also in higher dimensions, using methods based on the sub-additive ergodic theorem [1-4,8,11,12]. The continuous time case under the name stochastic combustion process was treated in $[13,14]$. It appears that the sub-additivity is completely lost when $M<\infty$. Our main purpose here is to develop new methods to study such models, and to go beyond methods based on sub-additivity. Also, we are particularly interested here in the fluctuations of the fronts. If $N_{t}$ represents the number of particles at the front $r_{t}$ at time $t$, then $r_{t}$ moves to the right at rate $N_{t}$, and hence $r_{t}-\int_{0}^{t} N_{s} \mathrm{~d} s$ is a martingale. The standard approach to the law of large numbers would then be to show that, as observed from the front, the system has an ergodic invariant measure $\mu$, and $t^{-1} \int_{0}^{t} N_{s} \mathrm{~d} s \rightarrow E_{\mu}[N]$. The central limit theorem would be proved by showing that the time correlations of $N_{s}$ decay fast enough. However, very few methods exist for proving uniqueness, or ergodicity, of invariant measures of such systems. Furthermore, although one can guess that the correlations of $N_{s}$ decay quickly - likely exponentially fast - it is not at all apparent how to prove it. The method we use to prove the law of large numbers and central limit theorem for the front, $r_{t}$, is that of regeneration times. Because the front moves ballistically while the particles move diffusively, one expects there are regeneration times after which the trajectory of the front is decoupled from that of the particles behind the front. Note that a concept similar to that of regeneration times, known as cluster structure, has been well know for many years in the context of mechanically interacting one-dimensional dynamical systems of particles [5] (also see [15] and references therein).

The combustion process that we consider is related to deterministic reaction-diffusion equations of the form $\partial_{t} u=$ $\partial_{x}^{2} u+f(u)$. The two key differences are the discreteness of the variable - we have a number of particles as opposed to a continuum variable $u$ - and the stochasticity. The effect of discreteness and/or stochasticity on the traveling waves of reaction-diffusion equations is a question that has not received the attention it deserves, as these effects are usually present in real systems. Therefore, we believe it is crucial to develop methods to study such systems. In the literature of reaction-diffusion equations one separates several cases according to the behavior of $f$ near zero. An $f$ which vanishes on $[0, \theta]$ for some $\theta>0$ with $f(u)>0$ for $u>\theta$ is said to have a combustion nonlinearity with ignition temperature cutoff $\theta$ (see, for example [7]). Note that the discreteness of the particle models of the type we consider makes them analogous to the combustion nonlinearity with the ignition temperature cutoff corresponding to a single particle.

In the second section of this article, we define the combustion process and state the main result (Theorem 1). In Section 3, we define an auxiliary and a labeled process, which will be needed to define the renewal structure leading to the regeneration times. The labeled process can be understood as a combustion process where particles are labeled so that if at a given site a particle has to be killed, it is the one with the smallest label. The auxiliary process moves ballistically to the right and is coupled to the labeled combustion process in such a way that it is always to the left of the right-most visited site $r_{t}$. In Section 4, these processes are used to define the renewal structure, and the corresponding regeneration times. Then in Section 5, it is proved that the regeneration times have finite moments of order 2, under appropriate assumption on the threshold $M$. In Section 6 we complete the proof of Theorem 1. 


\section{Combustion process}

We define a stochastic process describing the dynamics of particles on the lattice $\mathbb{Z}$ which move as rate 2 continuous time simple symmetric random walks and branch and are killed at rates depending on the configuration of neighboring particles. The branching and killing depend on natural number parameters $2 \leqslant a \leqslant M$. Each particle performs a continuous time symmetric simple random walk independent of the others, with two twists: There is a position $r \in \mathbb{Z}$ which is the rightmost visited site. When a particle jumps right from this site, it branches into $a$ particles, with the result that there are $a$ particles at a new rightmost visited site, $r+1$. In addition, we allow at most $M$ particles at any site, and maintain this requirement by killing any particle that attempts to jump to a site with $M$ particles.

The state space of our system is

$$
\Omega:=\left\{(r, \eta): r \in \mathbb{Z}, \eta \in\{0, \ldots, M\}^{\{\ldots, r-1, r\}}\right\} .
$$

Here $\eta(x)$ denotes the number of particles at $x \leqslant r$ and we will write $\eta_{t}(x)$ or $\eta(t, x)$ to denote the same quantity at time $t$.

The process is Markov with infinitesimal generator

$$
L f(r, \eta)=\sum_{x \leqslant r, y \leqslant r,|x-y|=1} \eta(x)\left(f\left(r, T_{x y} \eta\right)-f(r, \eta)\right)+\eta(r)\left(f\left(r+1, \eta-\delta_{r}+a \delta_{r+1}\right)-f(r, \eta)\right),
$$

where $\delta_{x}$ denotes the configuration with one particle at $x$ and

$$
T_{x y} \eta=\eta-\delta_{x}+\delta_{y} 1(\eta(y)<M) .
$$

In the following we will assume for technical reasons that

$$
8<M<\infty .
$$

We can now state the main results.

Theorem 1. Suppose the process is started with initial conditions $r=0, \eta(0,0) \in\{1, \ldots, M\}$ and $\eta(0, x) \in$ $\{0, \ldots, M\}$ arbitrary for $x<0$.

(i) (Law of large numbers). There exists a nonrandom $v \in(0, \infty)$, which does not depend on the initial condition $\{\eta(0, x): x \leqslant 0\}$, such that almost surely,

$$
\lim _{t \rightarrow \infty} \frac{r_{t}}{t}=v \text {. }
$$

(ii) (Central limit theorem). There exists a nonrandom $\sigma \in(0, \infty)$, which does not depend on the initial condition $\{\eta(0, x): x \leqslant 0\}$, such that

$$
\epsilon^{1 / 2}\left(r_{\epsilon^{-1} t}-\epsilon^{-1} v t\right), \quad t \geqslant 0,
$$

converges in law as $\epsilon \rightarrow 0$ to Brownian motion with variance $\sigma^{2}$.

To prove this theorem we will define a renewal structure for the right-most visited site $r_{t}$, in terms of regeneration times, and then will show that such times have finite second moments.

\section{Auxiliary and labeled processes}

In this section, we will define an auxiliary process and a labeled process, both coupled to the combustion process.

The auxiliary process $\left\{\tilde{r}_{t}: t \geqslant 0\right\}$, will take values on $\mathbb{Z}$ and will have two fundamental properties: Under appropriate initial conditions on the stochastic combustion process it will always be to the left of the right-most visited site $\left\{r_{t}: t \geqslant 0\right\}$, and its dynamics will be independent of all particles appearing to the left of $r_{0}$ in the stochastic combustion process. These properties will help us define a renewal structure for the right-most visited site $\left\{r_{t}: t \geqslant 0\right\}$ and will give a lower bound for the limiting speed $v$ of $r_{t}$. This is the content of Lemma 4. 
The labeled process has a state space larger than the combustion process, with particles carrying labels. An explicit rule for killing is given in which when a particle is killed, it is the one with the smallest label. We also introduce a variant of the labeled process, called the enlarged process, in which we keep track of the killed particles as well.

We now begin the constructions. Start with a set of independent continuous time rate 2 simple symmetric random walks $Y_{x, i}$, with label $(x, i)$, where $x \in \mathbb{Z}$ and $i \in\{1, \ldots, a-1\}$. Each random walk $Y_{x, i}, i \in\{1, \ldots, a-1\}$ starts at site $x$. We order the labels by

$$
(x, i)<\left(x^{\prime}, i^{\prime}\right) \quad \text { if } x<x^{\prime} \quad \text { or } x=x^{\prime} \text { and } i<i^{\prime} .
$$

\subsection{Auxiliary process}

Let $r \in \mathbb{Z}$ and for each $z \in \mathbb{Z}$, define $B_{z}$ as the set of $M$ largest labels not exceeding $(z, a-1)$ in the order (6). Define $A_{r, z}$ to be the labels in $B_{z}$ with $x \geqslant r$.

We define a sequence of waiting times. Let $v_{0}:=0$ and define $v_{1}$ as the first time one of the random walks $\left\{Y_{z, i}:(z, i) \in A_{r, r}\right\}$, hits the site $r+1$. Next, define $\nu_{2}$ as the first time one of the random walks $\left\{Y_{z, i}:(z, i) \in A_{r, r+1}\right\}$, hits the site $r+2$. In general, for $k \geqslant 3$, we define $v_{k}$ as the first time one of the random walks $\left\{Y_{z, i}:(z, i) \in A_{r, r+k-1}\right\}$, visits the site $r+k$. Finally, for $n \in \mathbb{N}$, let

$$
\tilde{r}_{t}^{r}:=r+n, \quad \text { if } \sum_{k=0}^{n} v_{k} \leqslant t<\sum_{k=0}^{n+1} v_{k},
$$

where the superscript $r$ in $\tilde{r}_{t}^{r}$ indicates that $\tilde{r}_{0}^{r}=r$.

Lemma 1. There exists an $\alpha>0$ which does not depend on $r$ such that a.s.

$$
\lim _{t \rightarrow \infty} \frac{\tilde{r}_{t}^{r}}{t}=\alpha
$$

Proof. Once one notes that for every $j \geqslant 0$ the random variables $\left\{v_{k \ell+j}: k \geqslant 1\right\}$ are independent whenever $\ell>M /(a-1)$, the proof is a simple exercise.

\subsection{Labeled process}

We enlarge the state space of the stochastic combustion process so that particles carry labels which tell us where they originated.

We will want to keep track of where particles came from, even after restarting at stopping times. Hence each particle will have a starting position $z \in \mathbb{Z}$ and label $(x, i), x \in \mathbb{Z}, i \in\{1, \ldots, a-1\}$ describing its birthplace. We will allow the possibility that $z \neq x$.

At time 0 , we have an $r \in \mathbb{Z}$ representing the rightmost visited site, and a subset $\mathcal{I}(0)$ of the labels $(x, i)$ with $x \leqslant r$, representing the set of labels of live particles at time 0 . To each one of these labels is assigned a position $z=Z_{x, i}(0) \leqslant r$ which is the position at time $t=0$ of that particle. The position at time $t$ is $Z_{x, i}(t)=Y_{x, i}(t)+z-x$.

To keep track of the killing in this process, we make a rule that whenever a particle jumps to a site with $M$ particles, the particle at that site with the smallest label is killed and the corresponding label is removed from the set $\mathcal{I}$ of labels of live particles.

The first time a particle jumps to site $r+1$, the labels $\{(r+1,1), \ldots,(r+1, a-1)\}$ corresponding to particles with initial positions $r+1$, are added to the set of labels of live particles. The time this happens will be denoted $\rho_{1}$. These particles then have trajectories $Z_{r+1, i}(t)$ which is equal to $Y_{r+1, i}\left(t-\rho_{1}\right)$ for $t \geqslant \rho_{1}$.

Similarly, for $k \geqslant 2, \rho_{1}+\cdots+\rho_{k}$ will be the first time a particle jumps to $r+k$ and at that time $\{(r+k, 1), \ldots$, $(r+k, a-1)\}$ are added to $\mathcal{I}$, with trajectories $Z_{r+k, i}(t)=Y_{r+k, i}\left(t-\rho_{1}-\cdots-\rho_{k}\right)$ for $t \geqslant \rho_{1}+\cdots+\rho_{k}$ until such time as the particles are killed and their label removed from the set of labels of live particles.

We denote by $\mathcal{I}(t)$ the set of labels of live particles at time $t$ and by $\mathcal{Z}(t)=\left\{Z_{x, i}(t):(x, i) \in \mathcal{I}(t)\right\}$ the positions of the corresponding random walks.

To avoid pathologies it is useful to insist that initially the set of labels of live particles includes at least one with $x=r$. The rightmost visited site $r_{t}$ is the supremum of the $x$ over the collection of labels, $r_{t}=\sup \{x:(x, i) \in \mathcal{I}(t)\}$. 
Let us formalize the above discussion. Call $\mathbb{L}$ the set of triples $(r, \mathcal{I}, \mathcal{Z})$ formed by an integer $r \in \mathbb{Z}$, a set of labels $\mathcal{I} \subset\{(x, i): x \leqslant r, 1 \leqslant i \leqslant a-1\}$ and position function $\mathcal{Z}: \mathcal{I} \rightarrow\{\ldots, r-2, r-1, r\}$ taking values in the integers smaller than or equal to $r$. We now define

$$
\mathbb{S}:=\left\{(r, \mathcal{I}, \mathcal{Z}) \subset \mathbb{L}: \max _{(x, i) \in \mathcal{I}} x=r, \max _{z \leqslant r} \sum_{(x, i) \in \mathcal{I}} \mathbf{1}_{\mathcal{Z}(x, i)}(z) \leqslant M\right\},
$$

which will be the state space of our process.

The labeled process starting from $w=(r, \mathcal{I}(0), \mathcal{Z}(0))$, is now defined as the triple $w_{t}=\left\{\left(r_{t}, \mathcal{I}(t), \mathcal{Z}(t)\right): t \geqslant 0\right\}$, defining a strong Markov process taking values on $\mathbb{S}$, and with a law given by a probability measure $\mathbb{P}_{w}$ defined on the Skorohod space $\mathcal{D}([0, \infty) ; \mathbb{S})$.

The combustion process described in Section 1 is the particle count $\eta(t):=\left\{\eta(t, y): y \leqslant r_{t}\right\}$ where $\eta(t, y)=$ $\sum_{(x, i) \in \mathcal{I}(t)} 1\left(Z_{x, i}(t)=y\right)$. We will occasionally use the more explicit notation $\eta_{w}(t)$ and $\eta_{w}(t, y)$ instead of $\eta(t)$ and $\eta(t, y)$ respectively, with the understanding that $\eta_{w}(0)$ is the particle count of the initial condition $w$.

We already defined $\rho_{1}$ to be the first time that one of these particles hits $r+1$.

Lemma 2. Suppose that $(r, 1), \ldots,(r, a-1) \in \mathcal{I}(0)$, and that all particles with these labels are initially at $r$. Then $\rho_{1} \leqslant v_{1}$.

Proof. The dynamics of the $a-1$ particles which start at $r$ is the same in the labeled process as in the ones we look at in the auxiliary process. Indeed, these are the only particles considered in the auxiliary process up to time $v_{1}$, while the labeled process may have many others, each of which has a chance to be the first to hit $r+1$.

Recall that $\rho_{k}+\cdots+\rho_{1}$ is the first time one of the labeled particles in the labeled process hits $r+k$. Note that the hitting could be done by one of the $a$ new particles created at $r+i$ at time $\rho_{i}, i<k$.

Lemma 3. Suppose that $(r, 1), \ldots,(r, a-1) \in \mathcal{I}(0)$, and that all particles with these labels are initially at $r$. Then $\rho_{k} \leqslant v_{k}$.

Proof. Before giving a general proof, we describe the special case of $M=a=k=2$ where the idea is more transparent.

Case $M=a=k=2$ : Note first of all that if $a=2$, we have $i=1$ always and hence we can drop the $i$ in the labels. The labeled process starts with one particle with label $r$ at $r$, possibly one other particle at site $r$, with a label smaller than $r$, and other particles with labels smaller than $r$ at arbitrary positions to the left of $r$. At time $\rho_{1}$ we have one particle labeled $r+1$ at $r+1$, which, after that time, has trajectory $Z_{r+1}(t)=Y_{r+1}\left(t-\rho_{1}\right)$, and another particle, labeled $r$ at some site $x \leqslant r+1$, which, after that time, has trajectory $Z_{r}(t)=Y_{r}(t)$. Note that neither particle can be killed before time $\rho_{1}+\rho_{2}$ because they are the two with the highest labels until that time. There could also be other particles with other labels at positions $x \leqslant r+1$. Denote by $\tau_{r+1}=\inf \left\{t \geqslant \rho_{1}: Z_{r+1}(t)=r+2\right\}$, by $\tau_{r}=$ $\inf \left\{t \geqslant \rho_{1}: Z_{r}(t)=r+2\right\}$ and by $\tau_{\text {others }}$ the first time one of the others hits $r+2$. Then $\rho_{2}=\min \left\{\tau_{r+1}, \tau_{r}, \tau_{\text {others }}\right\}-$ $\rho_{1} \leqslant \min \left\{\tau_{r+1}, \tau_{r}\right\}-\rho_{1}$.

On the other hand, $v_{2}=\min \left\{\sigma_{r}, \sigma_{r+1}\right\}$ where $\sigma_{r}=\inf \left\{t \geqslant 0: Y_{r}(t)=r+2\right\}$ and $\sigma_{r+1}=\inf \left\{t \geqslant 0: Y_{r+1}(t)=\right.$ $r+2\}$. Now

$$
\begin{aligned}
\tau_{r+1} & =\inf \left\{t \geqslant \rho_{1}: Z_{r+1}(t)=r+2\right\}=\inf \left\{t \geqslant \rho_{1}: Y_{r+1}\left(t-\rho_{1}\right)=r+2\right\} \\
& =\inf \left\{t \geqslant 0: Y_{r+1}(t)=r+2\right\}+\rho_{1}=\sigma_{r+1}+\rho_{1}
\end{aligned}
$$

and

$$
\begin{aligned}
\tau_{r} & =\inf \left\{t \geqslant \rho_{1}: Z_{r}(t)=r+2\right\}=\inf \left\{t \geqslant \rho_{1}: Y_{r}(t)=r+2\right\} \\
& \leqslant \inf \left\{t \geqslant 0: Y_{r}(t)=r+2\right\}+\rho_{1}=\sigma_{r}+\rho_{1} .
\end{aligned}
$$

The result follows.

General case: Let us examine the configuration in the labeled process at time $\rho_{1}+\cdots+\rho_{k-1}$. It has $a-1$ particles at site $r+k-1$ with labels $(r+k-1,1), \ldots,(r+k-1, a-1)$ plus one additional particle with an unknown label, the 
one which hit $r+k-1$. An additional $M-a+1$ particles (which might include the additional particle of unknown label which hit $r+k-1$ ) with the next highest labels after the first $a-1$ have some unknown positions, and there may in addition be any number of other particles in the configuration. In the time interval $\left[0, \rho_{1}+\cdots+\rho_{k-1}\right]$ none of the $M-a+1$ particles has hit $r+k$. Their trajectories in the time interval $\left[\rho_{1}+\cdots+\rho_{k-1}, \rho_{1}+\cdots+\rho_{k}\right]$ are $Y_{(r+k-2, a-1)}\left(t-\rho_{1}+\cdots+\rho_{k-2}\right), \ldots$ The definition of $v_{k}$, on the other hand, involves the particles with these $M$ leading indices, but without the time shift. Hence the first time that any of the first $a-1$ particles hits is identical to that of the auxiliary process, the first time that any of the next $M-a+1$ particles hits is greater in the auxiliary process, and one of the other possible particles in the labeled process could be the one which hit first, making the time shorter still.

\subsection{Enlarged process}

We have seen that the labeled process is defined in terms of a set of labels $\mathcal{I}(t)$, for $t \geqslant 0$, with the property $r_{t}=\sup \{x:(x, i) \in \mathcal{I}(t)\}<\infty$. Whenever a particle with a label in $\mathcal{I}(t)$ is killed, this label is removed. In the enlarged process we keep track of the killed particles as well. Define for each $t \geqslant 0$, the set of labels of all activated particles up to time $t$ in the labeled process,

$$
\overline{\mathcal{I}}(t)=\bigcup_{0 \leqslant s \leqslant t} \mathcal{I}(s)
$$

Consider the set $\overline{\mathcal{Z}}(t)=\left\{Z_{x, i}(t):(x, i) \in \overline{\mathcal{I}}(t)\right\}$ of all the corresponding random walks at time $t$. The enlarged process is defined as the triple $\bar{w}_{t}=\left\{\left(r_{t}, \overline{\mathcal{I}}(t), \overline{\mathcal{Z}}(t)\right): t \geqslant 0\right\}$. Observe that the particle count $(t, y) \mapsto$ $\sum_{(x, i) \in \overline{\mathcal{I}}(t)} 1\left(Z_{x, i}(t)=y\right)$ of the enlarged process, is the combustion process without threshold $(M=\infty)$.

Consider now the set of labels $\mathcal{R}(t)$, obtained after removing from $\mathcal{I}(t)$ all labels $(x, i)$ with $x<r=\sup \{y:(y, i) \in$ $\mathcal{I}(0)\}$. We define for $y \leqslant r_{t}$ the particle count

$$
\zeta(y, t)=\sum_{(x, i) \in \mathcal{R}(t)} 1\left(Z_{x, i}(t)=y\right) .
$$

Also let $\mathcal{L}(t)$ be the set of labels obtained after removing from $\mathcal{I}(t)$ all labels $(x, i) x \geqslant r$. We define for $y \leqslant r_{t}$ the particle count

$$
\phi(y, t)=\sum_{(x, i) \in \mathcal{L}(t)} 1\left(Z_{x, i}(t)=y\right) .
$$

We similarly define $\overline{\mathcal{L}}(t)$ as the set of labels obtained after removing from $\overline{\mathcal{I}}(t)$ all labels $(x, i)$ with positions $x \geqslant r$, and the corresponding particle count for $y \leqslant r_{t}$ as

$$
\bar{\phi}(y, t)=\sum_{(x, i) \in \overline{\mathcal{L}}(t)} 1\left(Z_{x, i}(t)=y\right) .
$$

Sometimes we will write $\zeta_{w}, \phi_{w}$ and $\bar{\phi}_{w}$ to emphasize the dependence on the initial condition $w \in \mathbb{S}$.

\section{Lemma 4.}

(i) For every initial condition $w \in \mathbb{S}$ and every $t \geqslant 0$ and $x \leqslant r_{t}$

$$
\phi_{w}(x, t) \leqslant \bar{\phi}_{w}(x, t) \text {. }
$$

(ii) For every $w=(r, \mathcal{I}(0), \mathcal{Z}(0)) \in \mathbb{S}$ with labels $(r, 1), \ldots,(r, a-1)$ at site $r$ and corresponding initial positions $Z_{r, i}(0)=r$ for $1 \leqslant i \leqslant a-1$,

$$
\tilde{r}_{t}^{r} \leqslant r_{t} \text {. }
$$

(iii) For every $w=(r, \mathcal{I}(0), \mathcal{Z}(0)) \in \mathbb{S}$, the processes $\bar{\phi}_{w}$ and $\tilde{r}_{t}^{r}$ are independent.

Proof. Part (i) follows directly from the definitions. Part (ii) is a consequence of Lemma 3. Part (iii) follows from the observation that $\bar{\phi}$ and $\tilde{r}_{t}^{r}$ are defined in terms of the random walks $\left\{Y_{x, i}: x<r\right\}$ and $\left\{Y_{x, i}: x \geqslant r\right\}$ respectively, which are independent. 


\section{The renewal structure}

At a heuristic level, regeneration occurs every time the front moves forward and the particles behind never catch it up later on. After such a time, the trajectory of the front depends only on the $a-1$ newly created particles sitting on the front, and the new particles they will create in the future.

In order to estimate the tails of regeneration times, it is useful to decouple particles initially on the front from particles to the left of it. We require that, from such a time on, the $a-1$ new particles on the front, and the ones they will create, move fast enough, and particles initially to the left do not move fast. The precise definition is given now in a sequence of definitions leading to (18).

Consider the enlarged process $\bar{w}_{t}$ and its natural filtration $\overline{\mathcal{F}}_{t}$, with an initial condition $w_{0}$ having particles with labels $(r, 1), \ldots,(r, a-1)$ at site $r$, and any allowable configuration of particles with labels to the left of $r$. Let $\alpha=\lim _{t \rightarrow \infty} \tilde{r}_{t} / t$ be as in (8) and choose any $0<\alpha^{\prime}<\alpha$. Define

$$
U:=\inf \left\{t \geqslant 0: \tilde{r}_{t}-r<\left\lfloor\alpha^{\prime} t+1 / 2\right\rfloor\right\}
$$

where $\lfloor x\rfloor$ denotes the greatest integer less than or equal to $x$.

Define $V$ as the first time one of the particles initially strictly to the left of $r$ hits $\left\{x: x>\left\lfloor\alpha^{\prime} t\right\rfloor+r\right\}$,

$$
V:=\inf \left\{t \geqslant 0: \sup _{x>\left\lfloor\alpha^{\prime} t\right\rfloor+r} \bar{\phi}_{w_{0}}(x, t)>0\right\} .
$$

$U$ and $V$ in $[0, \infty]$ are stopping times with respect to $\left\{\overline{\mathcal{F}}_{t}: t \geqslant 0\right\}$. Let

$$
D:=\min \{U, V\} \text {. }
$$

Note that when $\{D=\infty\}$, since $r_{t} \geqslant \tilde{r}_{t}$, the jumps in the front $r_{t}$ are never produced by particles initially strictly to the left of $r$. For each $y \in \mathbb{Z}$, let

$$
T_{y}:=\inf \left\{t \geqslant 0: r_{t} \geqslant y\right\}
$$

denote the hitting time of $y$ by the rightmost visited site in the labeled process $w_{t}$.

We will also need the first time $U$ and $V$ happen after time $s \geqslant 0$,

$$
\begin{aligned}
& U \circ \theta_{s}:=\inf \left\{t \geqslant 0: \tilde{r}_{t}^{r_{s}}-r_{s}<\left\lfloor\alpha^{\prime} t+1 / 2\right\rfloor\right\}, \\
& V \circ \theta_{s}:=\inf \left\{t \geqslant 0: \sup _{x>\left\lfloor\alpha^{\prime} t\right\rfloor+r_{s}} \bar{\phi}_{w_{s}}(x, t)>0\right\},
\end{aligned}
$$

and $D \circ \theta_{s}:=\min \left\{U \circ \theta_{s}, V \circ \theta_{s}\right\}$.

Choose an integer $L:=M$ and define sequences $\left\{S_{k}: k \geqslant 0\right\}$ and $\left\{D_{k}: k \geqslant 1\right\}$ of $\overline{\mathcal{F}}_{t}$-stopping times as follows. Start with $S_{0}=0$ and $R_{0}=r$. Then define

$$
S_{1}:=T_{R_{0}+L}, \quad D_{1}:=D \circ \theta_{S_{1}}+S_{1}, \quad R_{1}:=r_{D_{1}},
$$

and, for $k \geqslant 1$,

$$
S_{k+1}:=T_{R_{k}+L}, \quad D_{k+1}:=D \circ \theta_{S_{k+1}}+S_{k+1}, \quad R_{k+1}:=r_{D_{k+1}} .
$$

Note that these times are not necessarily finite and we make the convention that $r_{\infty}=\infty$. Similarly, we define $U_{k}:=$ $U \circ \theta_{S_{k}}+S_{k}$ and $V_{k}:=V \circ \theta_{S_{k}}+S_{k}$ for $k \geqslant 1$. Let

$$
K:=\inf \left\{k \geqslant 1: S_{k}<\infty, D_{k}=\infty\right\},
$$

and define the regeneration time,

$$
\kappa:=S_{K}
$$

Note that $\kappa$ is not a stopping time with respect to $\overline{\mathcal{F}}_{t}$.

Denote by $\mathcal{G}$ the information up to time $\kappa$, defined as the completion with respect to $\mathbb{P}_{w}$ of the smallest $\sigma$-algebra containing all sets of the form $\{\kappa \leqslant t\} \cap A, A \in \overline{\mathcal{F}}_{t}$.

In Section 5, we will show that $\mathbb{E}_{w}\left[\kappa^{2}\right]<\infty$ and hence, in particular

$$
\mathbb{P}_{w}[\kappa<\infty]=1 \text {. }
$$


Newly created particles (and the ones they will further create) start afresh from regeneration times, ignoring all other active particles.

Proposition 1. Let A be a Borel subset of $\mathcal{D}([0, \infty) ; \Omega)$. Then,

$$
\mathbb{P}_{w}\left[\tau_{-r_{\kappa}} \zeta(\kappa+\cdot) \in A \mid \mathcal{G}\right]=\mathbb{P}_{(a-1) \delta_{0}}[\eta(\cdot) \in A \mid U=\infty]
$$

where $(a-1) \delta_{0}$ denotes a configuration with $a-1$ particles at 0 and none anywhere else.

Proof. We have to show that for any $B \in \mathcal{G}$,

$$
\mathbb{P}_{w}\left[B,\left\{\tau_{-r_{\kappa}} \zeta(\kappa+\cdot) \in A\right\}\right]=\mathbb{P}_{w}[B] \mathbb{P}_{(a-1) \delta_{0}}[\eta(\cdot) \in A \mid U=\infty]
$$

Now, using (19),

$$
\begin{aligned}
\mathbb{P}_{w} & {\left[B,\left\{\tau_{-r_{k}} \zeta(\kappa+\cdot) \in A\right\}\right]=\mathbb{P}_{w}\left[\{\kappa<\infty\}, B,\left\{\tau_{-r_{k}} \zeta(\kappa+\cdot) \in A\right\}\right] } \\
& =\sum_{k=1}^{\infty} \mathbb{P}_{w}\left[\left\{S_{k}<\infty, D_{k}=\infty\right\}, B, \tau_{-r_{\kappa}} \zeta(\kappa+\cdot) \in A\right] \\
& =\sum_{k=1}^{\infty} \sum_{x \in \mathbb{Z}} \mathbb{P}_{w}\left[r_{S_{k}}=x, S_{k}<\infty, D_{k}=\infty, B, \tau_{-x} \zeta\left(S_{k}+\cdot\right) \in A\right] .
\end{aligned}
$$

From the definition of $\mathcal{G}$ there is an event $B_{k} \in \overline{\mathcal{F}}_{S_{k}}$ such that $B_{k}=B$ on $\kappa=S_{k}$. Therefore, we can continue developing (21) to obtain,

$$
\begin{aligned}
& =\sum_{k=0}^{\infty} \sum_{x \in \mathbb{Z}} \mathbb{P}_{w}\left[r_{S_{k}}=x, S_{k}<\infty, D_{k}=\infty, B_{k}, \tau_{-x} \zeta\left(S_{k}+\cdot\right) \in A\right] \\
& =\sum_{k, x} \mathbb{E}_{w}\left[\mathbf{1}_{r_{S_{k}}=x, S_{k}<\infty, B_{k}} \mathbb{P}_{w}\left[D_{k}=\infty, \tau_{-x} \zeta\left(S_{k}+\cdot\right) \in A \mid \overline{\mathcal{F}}_{S_{k}}\right]\right]
\end{aligned}
$$

where $\mathbb{E}_{w}$ is the expectation defined by $\mathbb{P}_{w}$. But on the events $S_{k}<\infty$ and $r_{S_{k}}=x$, we have by parts (i) and (ii) of Lemma 4, that

$$
\zeta_{w}\left(S_{k}+\cdot\right)=\eta_{(a-1) \delta_{x}}(\cdot)
$$

when $U_{k}=V_{k}=\infty$, and that $\eta_{(a-1) \delta_{x}}(\cdot)$ is independent of the configuration of particles initially to the left of $x$. Here, $(a-1) \delta_{x}$, is the configuration with $(a-1)$ particles at site $x$ with labels $(x, 1), \ldots,(x, a-1)$ and none elsewhere. Indeed, part (i) of Lemma 4, and the event $V_{k}=\infty$, imply that the particles with initial positions $z$ to the left of $x$, are never at the right of $\left\lfloor\alpha^{\prime} t\right\rfloor+x$. And part (ii) of Lemma 4, and the event $U_{k}=\infty$, imply that the front $r_{t}$ is never to the left of $\left\lfloor\alpha^{\prime} t+1 / 2\right\rfloor+x$. Hence, there is no effect of the particles initially to the left of $x$ on the front $r_{t}$, so that $\zeta_{w}\left(S_{k}+\cdot\right)=\eta_{(a-1) \delta_{x}}(\cdot)$. Then, (22) combined with the independence of $U_{k}$ and $V_{k}$ given $\overline{\mathcal{F}}_{S_{k}}$ by part (iii) of Lemma 4, the translation invariance, and the strong Markov property imply that on the events $S_{k}<\infty$ and $r_{S_{k}}=x$,

$$
\begin{aligned}
\mathbb{P}_{w}\left[U_{k}=\infty, V_{k}=\infty, \tau_{-x} \zeta\left(S_{k}+\cdot\right) \in A \mid \overline{\mathcal{F}}_{S_{k}}\right] & =\mathbb{P}_{w}\left[U_{k}=\infty, \tau_{-x} \eta(a-1) \delta_{x}(\cdot) \in A \mid \overline{\mathcal{F}}_{S_{k}}\right] \mathbb{P}_{w}\left[V_{k}=\infty \mid \overline{\mathcal{F}}_{S_{k}}\right] \\
& =\mathbb{P}_{(a-1) \delta_{0}}[U=\infty, \eta(\cdot) \in A] \mathbb{P}_{w}\left[V_{k}=\infty \mid \overline{\mathcal{F}}_{S_{k}}\right] .
\end{aligned}
$$

Summarizing, we have,

$$
\begin{aligned}
\mathbb{P}_{w} & {[\kappa<\infty] \mathbb{P}_{w}\left[B, \tau_{-r_{k}} \zeta(\kappa+\cdot) \in A\right] } \\
& =\mathbb{P}_{(a-1) \delta_{0}}[U=\infty, \eta(\cdot) \in A] \sum_{k, x} \mathbb{P}_{w}\left[V_{k}=\infty, r_{S_{k}}=x, S_{k}<\infty, B_{k}\right] .
\end{aligned}
$$

Letting $A=\Omega$ gives

$$
\mathbb{P}_{w}[\kappa<\infty] \mathbb{P}_{w}[B]=\mathbb{P}_{(a-1) \delta_{0}}[U=\infty] \sum_{k, x} \mathbb{P}_{w}\left[V_{k}=\infty, r_{S_{k}}=x, S_{k}<\infty, B_{k}\right] .
$$

(23) and (24) together imply (20). 
Now define $\kappa_{1} \leqslant \kappa_{2} \leqslant \cdots$ by $\kappa_{1}:=\kappa$ and for $n \geqslant 1$

$$
\kappa_{n+1}:=\kappa_{n}+\kappa\left(\bar{w}_{\kappa_{n}+.}\right) \text {. }
$$

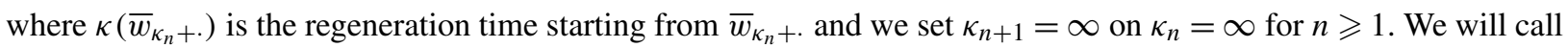
$\kappa_{1}$ the first regeneration time and $\kappa_{n}$ the $n$th regeneration time.

For each $n \geqslant 1$ the $\sigma$-algebra, $\mathcal{G}_{n}$ will be the completion with respect to $\mathbb{P}_{w}$ of the smallest $\sigma$-algebra containing all sets of the form $\left\{\kappa_{1} \leqslant t_{1}\right\} \cap \cdots \cap\left\{\kappa_{n} \leqslant t_{n}\right\} \cap A, A \in \mathcal{F}_{t_{n}}$. Clearly $\mathcal{G}_{1}=\mathcal{G}$.

Lemma 5. $\{U=\infty\} \in \mathcal{G}_{1}$.

Proof. Note that $\left\{\kappa_{1}=\infty\right\}$ is a null event for $\mathbb{P}_{w}$ and hence, since $\mathcal{G}_{1}$ is complete, it is enough to show that $\{U<\infty\} \cap$ $\left\{\kappa_{1}<\infty\right\} \in \mathcal{G}_{1}$.

For notational convenience, we write $\tilde{r}^{k}$ instead of $\tilde{r}^{r}{ }^{S_{k}}$. Note that whenever $U<\infty, S_{k}<\infty$ and $\tilde{r}_{U}>r_{S_{k}}$ happen, for some $k \geqslant 1$, then necessarily $\kappa_{1}>S_{k}$. Indeed, from the observation that $\tilde{r}_{S_{k}+.}=\tilde{r}_{\text {. }}^{k}$, we see that if the events $U<\infty, S_{k}<\infty$ and $\tilde{r}_{U} \geqslant r_{S_{k}}$ happen, then we must have that $U_{k}<\infty$, and hence $D_{k}<\infty$. By summing over the intersection with $\left\{\kappa_{1}=S_{k}\right\}$ we see that it follows that $\{U<\infty\} \cap\left\{\kappa_{1}<\infty\right\} \cap\left\{\tilde{r}_{U}>r_{\kappa_{1}}\right\}=\emptyset$. So

$$
\{U<\infty\} \cap\left\{\kappa_{1}<\infty\right\}=\left\{\tilde{r}_{U} \leqslant r_{\kappa_{1}}\right\} \cap\left\{\kappa_{1}<\infty\right\} .
$$

Since $\tilde{r}_{U} \leqslant r_{t}$ implies that $U<\infty$, it follows that

$$
\left\{\tilde{r}_{U}>r_{\kappa_{1}}\right\} \cap\left\{\tilde{r}_{U} \leqslant r_{t}\right\} \cap\left\{\kappa_{1} \leqslant t\right\}
$$

is empty. Hence

$$
\left\{\tilde{r}_{U} \leqslant r_{t}\right\} \cap\left\{\kappa_{1}<t\right\}=\left\{\tilde{r}_{U} \leqslant r_{\kappa_{1}}\right\} \cap\left\{\tilde{r}_{U} \leqslant r_{t}\right\} \cap\left\{\kappa_{1}<t\right\}=\left\{\tilde{r}_{U} \leqslant r_{\kappa_{1}}\right\} \cap\left\{\kappa_{1}<t\right\} .
$$

Thus

$$
\left\{\tilde{r}_{U} \leqslant r_{\kappa_{1}}\right\} \cap\left\{\kappa_{1}<\infty\right\}=\bigcup_{n=1}^{\infty}\left\{\tilde{r}_{U} \leqslant r_{n}\right\} \cap\left\{\kappa_{1}<n\right\} .
$$

The result then follows from the fact that $\left\{\tilde{r}_{U} \leqslant r_{t}\right\} \in \overline{\mathcal{F}}_{t}$ for each $t>0$ which is a direct consequence of the construction of the processes.

Proposition 2. Let A be a Borel subset of $\mathcal{D}([0, \infty) ; \Omega)$. Then,

$$
\mathbb{P}_{w}\left[\tau_{-r_{k n}} \zeta\left(\kappa_{n}+\cdot\right) \in A \mid \mathcal{G}_{n}\right]=\mathbb{P}_{(a-1) \delta_{0}}[\eta(\cdot) \in A \mid U=\infty] .
$$

Proof. Let $\psi: D[[0, \infty) ; \mathbb{S}) \rightarrow D[[0, \infty) ; \mathbb{S})$ be the map given by,

$$
\psi(\bar{w})(\cdot)=\tau_{-r_{\kappa_{1}}} \bar{w}\left(\kappa_{1}(\bar{w}(\cdot))+\cdot\right) .
$$

Then note that, $\mathcal{G}_{k+1}$ is generated by $\mathcal{G}_{1}$ and $\psi^{-1}\left(\mathcal{G}_{k}\right)$, and that the $\sigma$-algebras $\mathcal{G}_{1}$ and $\psi^{-1}\left(\mathcal{G}_{k}\right)$ are independent. The proof of this theorem now follows from the above observations, induction on $n \in \mathbb{Z}^{+}$using Proposition 1 , and Lemma 5.

We can now describe the full renewal structure.

Proposition 3. Let $w \in \mathbb{S}$. (i) Under $\mathbb{P}_{w}, \kappa_{1}, \kappa_{2}-\kappa_{1}, \kappa_{3}-\kappa_{2}, \ldots$ are independent, and $\kappa_{2}-\kappa_{1}, \kappa_{3}-\kappa_{2}, \ldots$ are identically distributed with law identical to that of $\kappa_{1}$ under $\mathbb{P}_{(a-1) \delta_{0}}[\cdot \mid U=\infty]$. (ii) Under $\mathbb{P}_{w}, r_{\cdot \wedge \kappa_{1}}, r_{\left(\kappa_{1}+\cdot\right) \wedge \kappa_{2}}-$ $r_{\kappa_{1}}, r_{\left(\kappa_{2}+\cdot\right) \wedge \kappa_{3}}-r_{\kappa_{2}}, \ldots$ are independent, and $r_{\left(\kappa_{1}+\cdot\right) \wedge \kappa_{2}}-r_{\kappa_{1}}, r_{\left(\kappa_{2}+\cdot\right) \wedge \kappa_{3}}-r_{\kappa_{2}}, \ldots$ are identically distributed with law identical to that of $r_{\kappa_{1}}$ under $\mathbb{P}_{(a-1) \delta_{0}}[\cdot \mid U=\infty]$.

Proof. This follows directly from Proposition 2. 


\section{Expectations and variances of the regeneration times}

\subsection{Estimates for the auxiliary process}

In this subsection we obtain some estimates for the auxiliary process. The process will always start from $(a-1) \delta_{0}$ and we denote the corresponding measure on the trajectories of the auxiliary process by $P$, and expectations by $E$.

Lemma 6. For $1 \leqslant p<M / 2$ and $j \geqslant M$, there exists a constant $C=C(p)<\infty$ such that

$$
E\left[\left|v_{j}\right|^{p}\right]<C \text {. }
$$

Proof. For $j \geqslant M$ the auxiliary process has its full complement of $M$ particles attempting to hit $j+1 . v_{j}$ is then the minimum of $\gamma_{1}, \ldots, \gamma_{M}$ which are the hitting times of 1 of $M$ random walks starting in $\{-M / a \leqslant x \leqslant 0\}$. Standard estimates for random walks give $P\left[\gamma_{i}>t\right] \leqslant C t^{-1 / 2}$ for some $C<\infty$. Hence $P\left[v_{j}>t\right] \leqslant C^{M} t^{-M / 2}$.

Lemma 7. For $1 \leqslant p<M / 2$, there exists a constant $C=C(p)<\infty$ such that for all initial conditions $w$, and all $t>0$

$$
P[t<U<\infty] \leqslant C t^{-(p / 2)} .
$$

Proof. By translation invariance we can assume $r=0$. Let $t_{1}$ be such that $\left\lfloor\alpha^{\prime} t_{1}+1 / 2\right\rfloor=M$. Then, when $t \geqslant t_{1}$, we have that

$$
P[t<U<\infty] \leqslant P\left[\tilde{r}_{t_{1}}^{0} \geqslant M, \bigcup_{s>t}\left\{\tilde{r}_{s}^{0}<\left\lfloor\alpha^{\prime} s+\frac{1}{2}\right\rfloor\right\}\right] .
$$

But, if $\tilde{r}_{s}^{0}<\left\lfloor\alpha^{\prime} s+1 / 2\right\rfloor$ for $s>t$, then $\sum_{j=1}^{\left\lfloor\alpha^{\prime} s+1 / 2\right\rfloor} v_{j}>s$, which in turn implies that, $\frac{1}{n} \sum_{j=1}^{n} v_{j} \geqslant \frac{1}{\alpha^{\prime}}\left(1-\frac{1}{2 n}\right)$ for some $n \geqslant\left\lfloor\alpha^{\prime} t+1 / 2\right\rfloor$. Similarly, $\tilde{r}_{t_{1}}^{0}>M$, implies that $\sum_{j=1}^{M} v_{j} \leqslant(M+1) / \alpha^{\prime}$. Therefore, whenever $\left\lfloor\alpha^{\prime} t+1 / 2\right\rfloor \geqslant$ $M+1$, we have

$$
\begin{aligned}
P[t<U<\infty] & \leqslant P\left[\sum_{j=1}^{M} v_{j} \leqslant \frac{M+1}{\alpha^{\prime}}, \bigcup_{n=\left\lfloor\alpha^{\prime} t+1 / 2\right\rfloor}^{\infty}\left\{\frac{1}{n} \sum_{j=1}^{n} v_{j} \geqslant \frac{1}{\alpha^{\prime}}\left(1-\frac{1}{2 n}\right)\right\}\right] \\
& \leqslant P\left[\bigcup_{n=\left\lfloor\alpha^{\prime} t+1 / 2\right\rfloor}^{\infty}\left\{\frac{1}{n} \sum_{j=M+1}^{n} v_{j} \geqslant \frac{1}{\alpha^{\prime}}\left(1-\frac{M+1 / 2}{n}\right)\right\}\right] .
\end{aligned}
$$

Now remark that for $j \geqslant M$, the random variables $v_{j}$ are identically distributed and have finite moments of order $p<M / 2$ by Lemma 6 . Each has expected value $1 / \alpha$. Define $\gamma_{j}=v_{j}-1 / \alpha$. Choose $t_{2}$ as any real number such that

$$
\beta=\frac{1}{\alpha^{\prime}}\left(1-\frac{M+1 / 2}{\left\lfloor\alpha^{\prime} t_{2}\right\rfloor}\right)-\frac{1}{\alpha}>0
$$

and $\left\lfloor\alpha^{\prime} t_{2}+1 / 2\right\rfloor \geqslant M+1$. Then, whenever $t \geqslant t_{2}$, if $N=\left\lfloor\alpha^{\prime} t+1 / 2\right\rfloor$, we have that,

$$
P[t<U<\infty] \leqslant P\left[\sup _{n \geqslant N} \frac{1}{n} \sum_{j=M+1}^{n} \gamma_{j} \geqslant \beta\right] .
$$

Recall that for each $0 \leqslant i<\ell, \ell=\lfloor M /(a-1)\rfloor+1$, the random variables $\left\{v_{k \ell+i}: k \geqslant 1\right\}$ are independent. Observe from (33) that, if $\beta^{\prime}=\beta a / M$,

$$
P[t<U<\infty] \leqslant \sum_{i=0}^{\ell-1} P\left[\sup _{n \geqslant N} \frac{1}{n} \sum_{j=M+1, j=k \ell+i}^{n} \gamma_{j} \geqslant \beta^{\prime}\right] .
$$

Suppose $X_{1}, X_{2}, \ldots$ are independent and identically distributed random variables with mean 0 . By Kolmogorov's inequality, 


$$
\begin{aligned}
P\left[\sup _{n \geqslant N} \frac{1}{n} \sum_{i=1}^{n} X_{i} \geqslant \epsilon\right] & \leqslant \sum_{k=0}^{\infty} P\left[\sup _{2^{k} N \leqslant n \leqslant 2^{k+1} N} \frac{1}{2^{k} N} \sum_{i=1}^{n} X_{i} \geqslant \epsilon\right] \\
& \leqslant \sum_{k=0}^{\infty}\left(2^{k} N \epsilon\right)^{-p} E\left[\left|\sum_{i=1}^{2^{k+1} N} X_{i}\right|^{p}\right] .
\end{aligned}
$$

Now, for $p \geqslant 2$, if $E\left[\left|X_{i}\right|^{p}\right]<\infty$ then $E\left[\left|\sum_{i=1}^{2^{k+1} N} X_{i}\right|^{p}\right] \leqslant C\left(2^{k+1} N\right)^{p / 2}$ for some $C<\infty$ (see item 16, page 60 of [10]), and hence for another $C<\infty$,

$$
P\left[\sup _{n \geqslant N} \frac{1}{n} \sum_{i=1}^{n} X_{i} \geqslant \epsilon\right] \leqslant C \epsilon^{-p} N^{-p / 2} .
$$

Applying (36) to (34), by Lemma 6 we obtain (30).

\subsection{Estimates for the enlarged process}

We start with a few standard estimates for hitting times of random walks.

Lemma 8. Let $\left\{X_{t}: t \geqslant 0\right\}$ be a continuous time simple symmetric random walk on $\mathbb{Z}$, of total jump rate 2 , starting from $x \leqslant 0, c>0$, and

$$
\tau:=\inf \left\{t \geqslant 0: X_{t}>\lfloor c t\rfloor\right\} .
$$

Then

$$
P[\tau=\infty] \geqslant \begin{cases}1-\exp \left\{x \theta_{c}\right\}, & x \leqslant-1, \\ \exp \{-2 / c\}\left(1-\exp \left\{-\theta_{c}\right\}\right), & x=0,\end{cases}
$$

where $\theta_{c}>0$ is the nonzero solution of $c \theta-2(\cosh \theta-1)=0$, and there exist $0<C, C^{\prime}<\infty$ such that

$$
P[t<\tau<\infty]<C^{\prime} \exp \{-C t\}\left(\exp \left\{-\frac{\theta_{c}|x+1|}{2}\right\}+\exp \left\{-I\left(\frac{|x+1|}{2 t}\right)\right\}\right),
$$

where $I(u)=2+u \sinh ^{-1}(u / 2)-\sqrt{4+u^{2}}$ is the rate function for the random walk.

Proof. For $\theta \in \mathbb{R}, \exp \left\{\theta X_{t}-2(\cosh \theta-1) t\right\}$ is a martingale. By the optional stopping time theorem,

$$
E\left[\exp \left\{\theta X_{\tau \wedge n}-2(\cosh \theta-1) \tau \wedge n\right\}\right]=\exp \{\theta x\} .
$$

Now, $X_{\tau \wedge n} \leqslant\lfloor c \tau \wedge n\rfloor+1$, hence $\theta X_{\tau \wedge n}-2(\cosh \theta-1) \tau \wedge n \leqslant(c \theta-2(\cosh \theta-1)) \tau \wedge n+\theta$. It follows that if $\theta \geqslant \theta_{c}$, we can apply the bounded convergence theorem in equality (39) taking the limit when $n \rightarrow \infty$, to conclude that,

$$
E\left[\mathbf{1}(\tau<\infty) \exp \left\{\theta X_{\tau}-2(\cosh \theta-1) \tau\right\}\right]=\exp \{\theta(x-1)\} .
$$

When $\tau<\infty$, we have $X_{\tau}=\lfloor c \tau\rfloor+1$ and therefore $\theta X_{\tau}-2(\cosh \theta-1) \tau \geqslant(c \theta-2(\cosh \theta-1)) \tau$. Letting $\theta \downarrow \theta_{c}$ in (40) and applying the bounded convergence theorem, we obtain,

$$
P[\tau<\infty] \leqslant \exp \left\{x \theta_{c}\right\} .
$$

To get the bound for $x=0$, note that the probability that the random walk does not move before time $t=1 / c$ is $\exp \{-2 / c\}$. Then use the Markov property and the result for $x=-1$.

To prove (38):

$$
P[t<\tau<\infty] \leqslant P\left[X_{t}>B\right]+P\left[t<\tau<\infty, X_{t} \leqslant B\right] .
$$

Now, whenever $B-x>0$,

$$
P\left[X_{t}>B\right] \leqslant \exp \left\{-t I\left(\frac{B+|x|}{t}\right)\right\}
$$


and by the strong Markov property, translation invariance and inequality (41),

$$
P\left[t<\tau<\infty, X_{t} \leqslant B\right] \leqslant P_{-(\lfloor c t\rfloor-B)}[\tau<\infty] \leqslant \exp \left\{-(\lfloor c t\rfloor-B) \theta_{c}\right\} .
$$

Choosing $B=(\lfloor c t\rfloor+x) / 2$, on the above inequalities, using the inequality $t I(u+v) \geqslant I(v)+(t-1) I(u)$ valid for $u, v \geqslant 0$, and substituting back in (42), gives (38).

Lemma 9. For each $\alpha^{\prime}<\alpha$, there exists $C<\infty$ such that for $t \geqslant 1$, and all $w \in \mathbb{S}$,

$$
\mathbb{P}_{w}[t<V<\infty] \leqslant C \exp \{-C t\} .
$$

Proof. Note that in the worst case in which there are $M$ random walks at the origin and at each site to the left of the origin, we get the bound,

$$
\mathbb{P}_{w}[t<V<\infty] \leqslant M \sum_{x=0}^{-\infty} P_{x}[t<\tau<\infty],
$$

where $\tau$ is defined in display (37). On the other hand, it is true that $\sum_{k=1}^{\infty} \mathrm{e}^{-I(k /(n+1))} \leqslant(n+1) \sum_{k=0}^{\infty} \mathrm{e}^{-I(k)}$. This estimate, the previous inequality and inequality (38) of Lemma 8 give us the result.

From Lemmas 7 and 9 we have

Corollary 1. For each $0 \leqslant p<M / 2$ there is a $C<\infty$ depending only on $p, M$ and $\alpha^{\prime}$ such that for all initial conditions $w$,

$$
\mathbb{P}_{w}[t<D<\infty] \leqslant C t^{-p / 2} .
$$

Lemma 10. There is a $\delta_{1}>0$ such that,

$$
\mathbb{P}_{w}[V<\infty]<1-\delta_{1} .
$$

Proof. Without loss of generality, $r=0$. Now, take the worst case scenario where $w$ has $M$ particles at each site $x \leqslant 0$. By Lemma 8 ,

$$
\mathbb{P}_{w}[V=\infty] \geqslant \mathrm{e}^{-2 M / \alpha^{\prime}}\left(1-\mathrm{e}^{-\theta_{c}}\right)^{M} \prod_{n=1}^{\infty}\left(1-\mathrm{e}^{-n \theta_{c}}\right)^{M}=\delta_{1}>0 .
$$

Lemma 11. Suppose that $M>4$. There is a $\delta_{2}>0$ such that for all initial conditions $w$ with at least a -1 particles at the rightmost visited site $r$

$$
\mathbb{P}_{w}[U<\infty]<1-\delta_{2} .
$$

Proof. We can also assume that $r=0$. To estimate $\mathbb{P}_{w}[U=\infty]$ below, note that

$$
\mathbb{P}_{w}[U=\infty]=P\left[\bigcap_{k=1}^{\infty}\left\{\sum_{j=1}^{k} v_{j} \leqslant \frac{2 k-1}{2 \alpha^{\prime}}\right\}\right] .
$$

Let $n \in \mathbb{N}$ and $0<\epsilon<1 /\left(2 \alpha^{\prime}\right)$ and define $G$ to be the event that each random walk $Y_{(x, i)}$ with $0 \leqslant x \leqslant n$, moves $M+1$ steps to the right before time $\epsilon$. When $G$ happens, $v_{k}<1 /\left(2 \alpha^{\prime}\right)$ and hence $\sum_{j=1}^{k} v_{j}<k /\left(2 \alpha^{\prime}\right)$ for all $k \leqslant$ $n+\lfloor M / a\rfloor:=n^{\prime}$. Note that $G \cap \bigcap_{k=1}^{\infty}\left\{\sum_{j=1}^{k} v_{j}<(2 k-1) / \alpha^{\prime}\right\} \supset G \cap H$ where

$$
H:=\bigcap_{k=n^{\prime}+1}^{\infty}\left\{\sum_{j=n^{\prime}+1}^{k} v_{j} \leqslant \frac{2 k-1}{2 \alpha^{\prime}}-n^{\prime} \epsilon\right\} .
$$


Furthermore, $G$ and $H$ are independent so $\mathbb{P}_{w}[U=\infty] \geqslant P[G] P[H]$. Now

$$
P\left[H^{c}\right] \leqslant \sum_{k=n^{\prime}+1}^{\infty} P\left[\sum_{j=n^{\prime}+1}^{k} v_{j} \geqslant \frac{2 k-1}{2 \alpha^{\prime}}-n^{\prime} \epsilon\right]
$$

and letting $\gamma_{j}=v_{j}-E\left[v_{j}\right]=v_{j}-1 / \alpha$, for $2 \leqslant p<M / 2$, with $\beta:=1 / \alpha^{\prime}\left(1-1 /\left(2 n^{\prime}\right)\right)-1 / \alpha>0$ when $n$ is large enough,

$$
P\left[\sum_{j=n^{\prime}+1}^{k} v_{j} \geqslant \frac{k}{\alpha^{\prime}}-n^{\prime} \epsilon\right] \leqslant E\left[\left(\sum_{j=n^{\prime}+1}^{k} \gamma_{j}\right)^{p}\right]\left(k \beta-n^{\prime} \epsilon\right)^{-p} \leqslant C k^{p / 2}\left(k \beta-n^{\prime} \epsilon\right)^{-p},
$$

where in the last inequality we used the same estimates explained between displays (35) and (36). Taking $\epsilon=\beta /\left(2 n^{\prime}\right)$ gives $k^{p / 2}\left(k \beta-n^{\prime} \epsilon\right)^{-p} \leqslant C^{\prime} k^{-p / 2}$. Hence $P\left[H^{c}\right] \leqslant C \sum_{k=n^{\prime}+1}^{\infty} k^{-p / 2}$ so as long as $p>2$ (which is possible since $M>4$ ) we obtain that $P\left[H^{c}\right]<1-\delta_{2}<1$ for sufficiently large $n$. Choose such an $n<\infty$, and note that for this $n$, $P[G] \geqslant \delta_{3}>0$ as well.

Lemma 12. Suppose that $M>4$. There is a $\delta>0$ such that for all initial conditions $w$ with at least a -1 particles at the rightmost visited site $r$

$$
\mathbb{P}_{w}[D<\infty]<1-\delta .
$$

Proof. Since $U$ and $V$ are independent by part (iii) of Lemma $4, \mathbb{P}_{w}[D<\infty]=1-\mathbb{P}_{w}[U=\infty] \mathbb{P}_{w}[V=\infty]$.

Lemma 13. There is a $C<\infty$ such that for every initial condition $w$ with $r=0$, and any $M^{\prime}>M$,

$$
\mathbb{P}_{w}\left[r_{t} \geqslant M^{\prime} t\right] \leqslant C \exp \{-C t\} .
$$

Proof. Note that $r_{t}$ is a process on $\mathbb{Z}$, increasing by one whenever a particle jumps to the right from $r$. Since there at most $M$ particles there, the maximum jump rate is $M$. The lemma then follows from standard estimates on Poisson processes.

Lemma 14. For each $p<M / 2$ there is a $C<\infty$ such that

$$
\mathbb{P}_{(a-1) \delta_{0}}\left[\kappa_{1}>t \mid U=\infty\right] \leqslant C t^{-p / 2} \text {. }
$$

Proof. Let us first write,

$$
\mathbb{P}_{(a-1) \delta_{0}}\left[\kappa_{1}>t \mid U=\infty\right]=\sum_{k=1}^{\infty} \mathbb{P}_{(a-1) \delta_{0}}\left[S_{k}>t, K=k \mid U=\infty\right]
$$

Applying recursively the strong Markov property to the stopping times $\left\{S_{j}: j \geqslant 1\right\}$ we see that for every $k \geqslant 1$,

$$
\mathbb{P}_{(a-1) \delta_{0}}\left[S_{k}>t, K=k \mid U=\infty\right] \leqslant(1-\delta)^{k-1},
$$

where $\delta>0$ is given by Lemma 12 . For any $\ell>0$ we therefore have,

$$
\mathbb{P}_{(a-1) \delta_{0}}\left[\kappa_{1}>t \mid U=\infty\right] \leqslant \sum_{k=1}^{\ell} \mathbb{P}_{(a-1) \delta_{0}}\left[t<S_{k}<\infty \mid U=\infty\right]+\delta^{-1}(1-\delta)^{\ell} .
$$

Let $1>\gamma>0$ and consider the event

$$
A_{k}=\left\{r_{D_{1}}-r_{S_{1}}<t^{\gamma}, r_{D_{2}}-r_{S_{2}}<t^{\gamma}, \ldots, r_{D_{k-1}}-r_{S_{k-1}}<t^{\gamma}\right\} .
$$

On $A_{k}$ we have $r_{S_{k}} \leqslant k\left(L+t^{\gamma}\right)$. Since $\tilde{r}_{t} \leqslant r_{t}$, if $U=\infty$, then $r_{t} \geqslant\left\lfloor\alpha^{\prime} t+1 / 2\right\rfloor$ for all $t>0$. Therefore, on $A_{k} \cap$ $\{U=\infty\}$,

$$
\left\lfloor\alpha^{\prime} S_{k}\right\rfloor \leqslant\left\lfloor\alpha^{\prime} S_{k}+1 / 2\right\rfloor \leqslant k\left(L+t^{\gamma}\right) .
$$


Hence for $t>\left(\ell\left(L+t^{\gamma}\right)+1\right) / \alpha^{\prime}$ and $k \leqslant \ell$,

$$
\mathbb{P}_{(a-1) \delta_{0}}\left[t<S_{k}<\infty, A_{k} \mid U=\infty\right]=0
$$

and therefore

$$
\mathbb{P}_{(a-1) \delta_{0}}\left[t<S_{k}<\infty \mid U=\infty\right] \leqslant \mathbb{P}_{(a-1) \delta_{0}}\left[A_{k}^{c}, S_{k}<\infty \mid U=\infty\right] .
$$

By Lemma 11, since $\mathbb{P}_{(a-1) \delta_{0}}[U=\infty] \geqslant \delta_{2}>0$. So for some $C<\infty$, the right-hand side of (52) is bounded above by

$$
C \sum_{i=1}^{k-1} \mathbb{P}_{(a-1) \delta_{0}}\left[r_{D_{i}}-r_{S_{i}} \geqslant t^{\gamma}, S_{k}<\infty\right] .
$$

Now let $M^{\prime}>M$ and

$$
\begin{aligned}
\mathbb{P}_{(a-1) \delta_{0}}\left[r_{D_{i}}-r_{S_{i}} \geqslant t^{\gamma}, S_{k}<\infty\right]= & \mathbb{P}_{(a-1) \delta_{0}}\left[r_{D_{i}}-r_{S_{i}} \geqslant t^{\gamma}, S_{k}<\infty, D_{i}-S_{i} \leqslant \frac{t^{\gamma}}{M^{\prime}}\right] \\
& +\mathbb{P}_{(a-1) \delta_{0}}\left[r_{D_{i}}-r_{S_{i}} \geqslant t^{\gamma}, S_{k}<\infty, D_{i}-S_{i}>\frac{t^{\gamma}}{M^{\prime}}\right] \\
\leqslant & \mathbb{P}_{(a-1) \delta_{0}}\left[r_{S_{i}+t^{\gamma} / M^{\prime}}-r_{S_{i}} \geqslant t^{\gamma}\right]+\mathbb{P}_{(a-1) \delta_{0}}\left[\frac{t^{\gamma}}{M^{\prime}}<D_{i}-S_{i}<\infty\right] .
\end{aligned}
$$

Note that in the last equation we used the fact that $S_{k}<\infty$ implies that $D_{i}-S_{i}<\infty$ for $i<k$. By the strong Markov property and Lemma 13,

$$
\mathbb{P}_{(a-1) \delta_{0}}\left[r_{S_{i}+t^{\gamma} / M^{\prime}}-r_{S_{i}} \geqslant t^{\gamma}\right] \leqslant C \exp -C t^{\gamma} .
$$

By the strong Markov property and Corollary 1 , for each $p<M / 2$,

$$
\mathbb{P}_{(a-1) \delta_{0}}\left[\frac{t^{\gamma}}{M^{\prime}}<D_{i}-S_{i}<\infty\right] \leqslant C t^{-\gamma p / 2} .
$$

Choosing $\ell=C \log t$ with $C=p\left(2 \log (1-\delta)^{-1}\right)^{-1}$ from (50) and the previous estimates we obtain (49).

Corollary 2. Let $\kappa_{1}$ be the first regeneration time of the stochastic combustion process.

(a) For $M>4$, $\mathbb{E}_{(a-1) \delta_{0}}\left[\kappa_{1} \mid U=\infty\right]<\infty$, and $\mathbb{E}_{(a-1) \delta_{0}}\left[r_{\kappa_{1}} \mid U=\infty\right]<\infty$.

(b) For $M>8, \mathbb{E}_{(a-1) \delta_{0}}\left[\kappa_{1}^{2} \mid U=\infty\right]<\infty$, and $\mathbb{E}_{(a-1) \delta_{0}}\left[r_{\kappa_{1}}^{2} \mid U=\infty\right]<\infty$.

Proof. The statements for $\kappa_{1}$ follow from Lemma 14, and those for $r_{\kappa_{1}}$ from Lemmas 13 and 14.

\section{Limit theorems}

In this section we use the renewal structure to prove Theorem 1, the law of large numbers and the central limit theorem for $r_{t}$. The argument for the law of large numbers follows that of Sznitman and Zerner in [17], developed in the context of multi-dimensional transient random walks in random environments. The argument for the central limit theorem is from [16].

\subsection{Law of large numbers}

We consider the stochastic combustion process started with an initial condition $r=0, \eta(0,0) \in\{1, \ldots, M\}$ and $\eta(0, x) \in\{0, \ldots, M\}$. We will prove that a.s.

$$
\lim _{t \rightarrow \infty} \frac{r_{t}}{t}=v:=\frac{\mathbb{E}_{(a-1) \delta_{0}}\left[r_{\kappa_{1}} \mid U=\infty\right]}{\mathbb{E}_{(a-1) \delta_{0}}\left[\kappa_{1} \mid U=\infty\right]}
$$


Let us first note that by Proposition 3, we have a.s.,

$$
\lim _{n \rightarrow \infty} \frac{\kappa_{n}}{n}=\mathbb{E}_{(a-1) \delta_{0}}\left[\kappa_{1} \mid U=\infty\right], \quad \text { and } \quad \lim _{n \rightarrow \infty} \frac{r_{\kappa_{n}}}{n}=\mathbb{E}_{(a-1) \delta_{0}}\left[r_{\kappa_{1}} \mid U=\infty\right] .
$$

For $t \geqslant 0$, define $n_{t}:=\sup \left\{n \geqslant 0: \kappa_{n} \leqslant t\right\}$, with the convention that $\kappa_{0}=0$. Note that (56) ensures that $n_{t}<\infty$ a.s. and by definition we also have, $\kappa_{n_{t}} \leqslant t<\kappa_{n_{t}+1}$, and $\lim _{t \rightarrow \infty} \kappa_{n_{t}}=\infty$. It follows from this inequality and the limit (56) that, a.s., $\lim _{t \rightarrow \infty} n_{t} / t=1 / \mathbb{E}_{(a-1) \delta_{0}}\left[\kappa_{1} \mid U=\infty\right]$ and hence almost surely,

$$
\lim _{t \rightarrow \infty} \frac{r_{\kappa_{n_{t}}}}{t}=\lim _{t \rightarrow \infty} \frac{r_{\kappa_{n_{t}}}}{\kappa_{n_{t}}} \frac{\kappa_{n_{t}}}{t}=v,
$$

Now

$$
\lim _{t \rightarrow \infty} \frac{\left|r_{t}-r_{\kappa_{n_{t}}}\right|}{t} \leqslant \lim _{t \rightarrow \infty} \frac{r_{\kappa_{n_{t}+1}}-r_{\kappa_{n_{t}}}}{t}=0 .
$$

from (57). This proves the law of large numbers.

\subsection{Central limit theorem}

Starting with the same initial conditions we consider

$$
B_{t}^{\epsilon}:=\epsilon^{1 / 2}\left(r_{\epsilon^{-1} t}-\epsilon^{-1} v t\right), \quad t \geqslant 0 .
$$

Define

$$
R_{j}:=r_{\kappa_{j+1}}-r_{\kappa_{j}}-\left(\kappa_{j+1}-\kappa_{j}\right) v, \quad j \geqslant 0,
$$

and denote for $m \geqslant 0$ the partial sums $\Sigma_{m}:=\sum_{j=1}^{m} R_{j}$.

For any $0 \leqslant t \leqslant T<\infty$,

$$
\left|B_{t}^{\epsilon}-\epsilon^{1 / 2} \Sigma_{n_{t / \epsilon}}\right| \leqslant 2 \epsilon^{1 / 2} \sup _{0 \leqslant n \leqslant n_{\left\lfloor\epsilon^{-1} T\right\rfloor}}\left(r_{\kappa_{n+1}}-r_{\kappa_{n}}\right)+2 v \epsilon^{1 / 2} \sup _{0 \leqslant n \leqslant n_{\left\lfloor\epsilon^{-1} T\right\rfloor}}\left(\kappa_{n+1}-\kappa_{n}\right) .
$$

For every $u>0$ we have, by Proposition 3

$$
\begin{aligned}
& \mathbb{P}_{(a-1) \delta_{0}}\left[\sup _{0 \leqslant n \leqslant n_{\left\lfloor\epsilon^{-1} T\right\rfloor}} \epsilon^{1 / 2}\left(\kappa_{n+1}-\kappa_{n}\right)>u\right] \\
& \quad \leqslant \mathbb{P}_{(a-1) \delta_{0}}\left[\kappa_{1}>\epsilon^{-1 / 2} u\right]+u^{-2} \epsilon\left(t \epsilon^{-1}+1\right) \mathbb{E}_{(a-1) \delta_{0}}\left[\kappa_{1}^{2} 1\left(\kappa_{1}>\epsilon^{-1 / 2} u\right) \mid U=\infty\right],
\end{aligned}
$$

which by part (b) of Corollary 2, a.s. converges to 0 as $\epsilon \rightarrow 0$. Hence, in probability

$$
\sup _{0 \leqslant n \leqslant n_{\left\lfloor\epsilon^{-1} T\right\rfloor}} \epsilon^{1 / 2}\left(\kappa_{n+1}-\kappa_{n}\right) \rightarrow 0
$$

and similarly

$$
\sup _{0 \leqslant n \leqslant n_{\left\lfloor\epsilon^{-1} T\right\rfloor}} \epsilon^{1 / 2}\left(r_{\kappa_{n+1}}-r_{\kappa_{n}}\right) \rightarrow 0 .
$$

Hence, $B_{t}^{\epsilon}-\epsilon^{1 / 2} \Sigma_{\epsilon_{\epsilon^{-1} t}}$ converges to 0 in probability, uniformly on compact sets of $t$. From Donsker's invariance principle, we know that $\sqrt{\epsilon} \Sigma_{\cdot / \epsilon}$ converges in law to a Brownian motion with variance $\mathbb{E}_{(a-1) \delta_{0}}\left[\left(r_{\kappa_{1}}-\right.\right.$ $\left.\kappa_{1} v\right)^{2} \mid U=\infty$ ], where $\Sigma_{s}, s \geqslant 0$, now stands for the linear interpolation of $\Sigma_{m}, m \geqslant 0$. From the previous proof we have $\lim _{t \rightarrow \infty} n_{t} / t=1 / \mathbb{E}_{(a-1) \delta_{0}}\left[\kappa_{1} \mid U=\infty\right]$. Since $\epsilon^{-1} k_{t \epsilon^{-1}}$ is increasing in $t$, the convergence is uniform on compact sets of $t$. This, together with the convergence in law of $\epsilon^{1 / 2} \Sigma_{. / \epsilon}$ to a Brownian motion with variance $\mathbb{E}_{(a-1) \delta_{0}}\left[\left(r_{\kappa_{1}}-\kappa_{1} v\right)^{2}\right]$, implies that $\epsilon^{1 / 2} \Sigma_{k_{\left\lfloor\epsilon^{-1} t\right\rfloor}}$ is tight in the Skorohod topology, and that its finite-dimensional distributions converge to the finite-dimensional distribution of a Brownian motion with variance,

$$
\sigma^{2}:=\frac{\mathbb{E}_{(a-1) \delta_{0}}\left[\left(r_{\kappa_{1}}-\kappa_{1} v\right)^{2} \mid U=\infty\right]}{\mathbb{E}_{(a-1) \delta_{0}}\left[\kappa_{1} \mid U=\infty\right]}
$$




\subsection{Non-degeneracy of the variance}

It suffices to prove that, for some $\alpha^{\prime}<\beta<v$,

$$
\mathbb{P}_{(a-1) \delta_{0}}\left[r_{\kappa_{1}}=L, L \beta^{-1} \leqslant \kappa_{1} \mid U=\infty\right]>0 .
$$

Now,

$$
\mathbb{P}_{(a-1) \delta_{0}}\left[r_{\kappa_{1}}=L, L \beta^{-1} \leqslant \kappa_{1}, U=\infty\right] \geqslant \mathbb{P}_{(a-1) \delta_{0}}\left[L \beta^{-1}<S_{1}<U, D \circ \theta_{S_{1}}=\infty\right] .
$$

The right-hand side we can write as

$$
\mathbb{E}_{(a-1) \delta_{0}}\left[1\left(L \beta^{-1}<S_{1}<U\right) \mathbb{E}_{(a-1) \delta_{0}}\left[1\left(V \circ \theta_{S_{1}}=\infty\right) 1\left(U \circ \theta_{S_{1}}=\infty\right) \mid \mathcal{F}_{S_{1}}\right]\right] .
$$

Given $\mathcal{F}_{S_{1}}, V \circ \theta_{S_{1}}$ and $U \circ \theta_{S_{1}}$ are independent, so

$$
\begin{aligned}
& \mathbb{E}_{(a-1) \delta_{0}}\left[1\left(V \circ \theta_{S_{1}}=\infty\right) 1\left(U \circ \theta_{S_{1}}=\infty\right) \mid \mathcal{F}_{S_{1}}\right] \\
& \quad=\mathbb{P}_{(a-1) \delta_{0}}\left[V \circ \theta_{S_{1}}=\infty \mid \mathcal{F}_{S_{1}}\right] \mathbb{P}_{(a-1) \delta_{0}}\left[U \circ \theta_{S_{1}}=\infty \mid \mathcal{F}_{S_{1}}\right] .
\end{aligned}
$$

But since by Lemmas 10 and 11 we have

$$
\mathbb{P}_{(a-1) \delta_{0}}\left[U \circ \theta_{S_{1}}=\infty \mid \mathcal{F}_{S_{1}}\right]=\mathbb{P}_{(a-1) \delta_{0}}[U=\infty] \geqslant \delta_{2}>0
$$

and

$$
\mathbb{P}_{(a-1) \delta_{0}}\left[V \circ \theta_{S_{1}}=\infty \mid \mathcal{F}_{S_{1}}\right] \geqslant \delta_{1}>0
$$

we have,

$$
\mathbb{P}_{(a-1) \delta_{0}}\left[L \beta^{-1}<S_{1}<U, D \circ \theta_{S_{1}}=\infty\right] \geqslant \delta_{1} \delta_{2} \mathbb{P}_{(a-1) \delta_{0}}\left[L \beta^{-1}<S_{1}<U\right] .
$$

But it is easy to check that $\mathbb{P}_{(a-1) \delta_{0}}\left[L \beta^{-1}<S_{1}<U\right]>0$. In fact, it is enough to lower bound this probability by the probability that one of the random walks at site 0 moves to site $L$ in a time $t$ such that $L \beta^{-1}<t<L\left(\alpha^{\prime}\right)^{-1}$, and then stays at site $L$ between time $t$ and time $L\left(\alpha^{\prime}\right)^{-1}$, while all other random walks between sites 0 and $L$ do not move at all during the time interval $\left[0, L\left(\alpha^{\prime}\right)^{-1}\right]$.

\section{References}

[1] O. Alves, F. Machado, S. Popov, Phase transition for the frog model, Electron. J. Probab. 7 (2) (2002).

[2] O. Alves, F. Machado, S. Popov, The shape theorem for the frog model, Ann. Appl. Probab. 12 (2) (2002) 533-546.

[3] O. Alves, F. Machado, S. Popov, K. Ravishankar, The shape theorem for the frog model with random initial configuration, Markov Process. Related Fields 7 (4) (2001) 525-539.

[4] O. Alves, C.E. Ferreira, F.P. Machado, Estimates for the spreading velocity of an epidemic model, Math. Comput. Simulation 64 (6) (2004) 609-616.

[5] C. Boldrighini, A. Pellegrinotti, E. Presutti, Ya.G. Sinai, M.R. Soloveichik, Ergodic properties of a semi-infinite one-dimensional system of statistical mechanics, Commun. Math. Phys. 101 (1985) 363-382.

[6] F. Comets, O. Zeitouni, A law of large numbers for random walks in random mixing environments, Ann. Probab. 32 (1B) (2004) 880-914.

[7] P.C. Fife, Mathematical Aspects of Reacting and Diffusing Systems, Lecture Notes in Biomath., vol. 28, Springer-Verlag, New York, 1979.

[8] L.R. Fontes, F.P. Machado, A. Sarkar, The critical probability for the frog model is not a monotonic function of the graph, J. Appl. Probab. 41 (1) (2004) 292-298.

[9] H. Kesten, A renewal theorem for random walk in a random environment, Proc. Sympos. Pure Math. 31 (1977) 67-77.

[10] V. Petrov, Sums of Independent Random Variables, Springer-Verlag, 1975.

[11] S. Popov, Frogs in random environment, J. Statist. Phys. 102 (1-2) (2001) 191-201.

[12] S. Popov, Frogs and some other interacting random walks models, Discrete Math. Theor. Comput. Sci. Proc., volume on Discrete Random Walks (2003), pp. 277-288 (electronic).

[13] A.F. Ramirez, V. Sidoravicius, Asymptotic behavior of a stochastic growth process associated with a system of interacting branching random walks, C. R. Math. Acad. Sci. Paris, Ser. I 335 (10) (2002) 821-826.

[14] A.F. Ramirez, V. Sidoravicius, Asymptotic behavior of a stochastic combustion growth process, J. Eur. Math. Soc. 6 (3) (2004) $293-334$.

[15] V. Sidoravicius, L. Triolo, M.E. Vares, Mixing properties for mechanical motion of a charged particle in a random medium, Commun. Math. Phys. 219 (2) (2001) 323-355.

[16] A.S. Sznitman, Slowdown estimates and central limit theorem for random walk in random environment, J. Eur. Math. Soc. 2 (2000) $93-143$.

[17] A.S. Sznitman, M. Zerner, A law of large numbers for random walks in random environment, Ann. Probab. 27 (4) (1999) $1851-1869$. 